\title{
The Effect of Platelet-Rich Fibrin on Donor Site of Split Thickness Skin Graft in Burned Patients
}

\author{
MINA R.S. SENADA, M.B.B.Ch.*; SAMEH M.E. ABD El RAHMAN, M.D.*; MOHAMED A.A. SALEH, M.D.*; \\ GHADA G. HAMAM, M.D.** and NIVEEN F. Al-MAHMOUDY, M.D.*
}

The Department of Plastic, Burn \& Maxillofacial Surgery* and The Department of Histology \& Cell Biology**, Faculty of Medicine, Ain Shams University, Cairo, Egypt

\begin{abstract}
Background: Patients with massive burn areas have now a greater chance of survival thanks to improved modalities of new treatments. In some patients it is necessary to harvest multiple skin grafts from the same donor site which is restricted by the quality of the skin and the time required to recover; this procedure can take a long time and may increase the danger of bacterial contamination resulting in increased morbidity and mortality in these individuals. Platelet-rich fibrin is one of the newest autologous growth factors utilized for wound management. PRF application can significantly improve the wounds' condition by supplying growth factors to the wound environment, which aid in the epithelialization process's acceleration, resulting in cost-effective wound management. This will allow multiple harvesting from the same donor site, reducing costs, hospitalization time, scars, and co-morbidities.
\end{abstract}

Aim of the Work: To assess the role of PRF in accelerating wound healing in the donor site following skin grafting in burn patients.

Patients and Methods: This prospective, controlled, clinical trial study was done from April 2020 to April 2021 included 20 patients at the burn unit of El Demerdash Hospital, Ain Shams University.

Results: The current study showed that healing days in donor sites injected with PRF were lower than those of controlled areas with statistical significance and mean epidermal thickness was lower in the control area compared to PRF injected areas with statistical significance.

Conclusion: PRF can significantly decrease the time of donor wound healing compared to that of conventional treatment.

Key Words: Donor site - Platelet-rich fibrin - Burn patients.

\section{INTRODUCTION}

The modality of soft tissue wound management within the last years has changed to keep the wound dry and clean which is important for the wound healing process. However, recently, there are more active factors that accelerate this process. Tissue engineering has resulted in the development of several new biological and clinical products that may have an impact on the wound healing process [1]. Applying those products may help in limiting the number of daily visits to healthcare hospitals, accelerating wound healing, and lowering treatment costs. The donor site of a split-thickness skin graft (STSG) must be carefully managed because patients frequently complain of more pain at the donor site so optimal donor site care would end in best healing. Major growth factors such as vascular endothelial growth factor (VEGF), platelet-derived growth factor (PDGF), insulin-like growth factor, and transforming growth factor (TGF) are abundant in platelets [2]. These growth factors can stimulate cell proliferation, matrix formation, and angiogenesis. Platelet-rich plasma (PRP) and Platelet-rich fibrin (PRF) are both considered sources of many growth factors that aid in soft tissue healing. PRF was initially used in oral implantology and was thought to be a healing biomaterial [3]. PRF has fibrin features with a distribution of platelet that mimics the body's response to the wound. The procedure uses a three-dimensional fibrin matrix that serves as a scaffold for platelet clot formation and growth factor collection (scaffolding). Scaffolding aids in the localization of growth factors, which aids tissue regeneration. The usage of PRF provides several advantages, including increased gene expression and protein production. Platelet activation is also influenced by differentiation factors [4]. These factors have a significant role in cellular activities like mitogenesis, chemotaxis, differentiation, and metabolism, as well as regulating and stimulating the healing process [5]. However, to date, only a few studies have looked into the impact of PRF in wound healing for donor sites of STSG [6]. As a result, our goal was to assess the role of PRF in accelerating wound healing in the donor site following skin grafting in burn patients. 


\section{PATIENTS AND METHODS}

This prospective controlled clinical trial was performed at the burn unit of Al Demerdash Hospital Ain Shams University from April 2020 to April 2021. Patients' informed written consent to participate in the study was signed before patient's enrollment.

A total of 20 patients were involved in our research. Patients with $2^{\text {nd }}$ or $3^{\text {rd-degree burns, }}$ ranging in age from 5 to 50 years, were included in our study. Patients with co-morbidities that interfere with the healing process, such as diabetes, liver cell failure, renal failure, and heart failure, were excluded.

\section{Platelet-rich fibrin preparation during operation:}

In the operation room, the plastic surgeon harvested STSG from the patient's thigh. To obtain the correct quantity and quality of fibrin matrix, platelets, and growth factors, a conventional PRF preparation methodology was followed. Autologous blood was collected in plastic tubes with no anticoagulants added, then centrifuged for 3 minutes at $700 \mathrm{rpm}$. The use of plastic tubes has a hydrophobic surface that does not effectively activate the coagulation process. As a result, all of the clotting factors and platelets of blood essential for platelet concentrate synthesis reach the upper zone of the tube under centrifugal force within the first 2-4 minutes. These separated plasma and platelets, which are situated in the upper layer and are pale yellow, are used in an injectable form [7]. It is important to shorten the time between blood sample preparation and centrifugation process as PRF remains in fluid form for 15 minutes before it is converted to a gel-like membrane. Then donor site of STSG was injected with PRF in the upper half (proximal part) and the lower half was considered as a control.

\section{Post-operative follow-up:}

Dressings were done on day 10 and day 14 after surgery (Fig. 3). On day 14 punch biopsy was done under local anesthesia from both the untreated donor site (control) and PRF treated area. Specimens were immediately fixed in $10 \%$ neutral buffered formalin for 24 hours, then were dehydrated in ascending grades of alcohol as follows: $95 \%$ ethanol for 3 changes, the first 2 changes for 1 hour each, and the last change for 2 hours then in absolute alcohol for another 3 changes, then the specimens were cleared in xylene. After that, they were then immersed in two changes of paraffin for one hour each [8]. Then, after obtaining paraffin blocks, serial slices of $5 \mathrm{~mm}$ thickness were obtained and stained with Hematoxylin and Eosin (H\&E), Masson's trichrome, and Orcein.

The Leica DM2500 microscope with a built-in camera was used to examine histological sections (Wetzlar, Germany). All photos were digitally recorded on a computer at Ain Shams University's Faculty of Medicine's Histology and Cell Biology Department. Measurements of mean epidermal thickness were acquired from three distinct slides obtained from each specimen.

\section{Ethical consideration:}

Local Ethical Committee at Ain Shams University's approval and patient's informed written consent to participate in the study were obtained from all participants in the study after explaining the study purpose, methods, risks and benefits. All patients' data were confidential, and the patient would not be mentioned by name in any published paper. All the participants in this study were allowed to withdraw at any time during study period. There are no financial conflicts of interest to disclose.

\section{Statistical analysis:}

Quantitative (numerical) normally distributed variables were described as mean \pm standard deviation (SD) and qualitative (categorical) data were described as numbers and percentages. The differences were statistically analyzed using the student$t$-test. Version 21 of the SPSS program was used to conduct the research (IBM Corporation, Somers, New York, USA). If the $p$-value was less than 0.05, the calculations were judged significant.

\section{RESULTS}

Baseline data of enrolled patients are shown in Table (1). The mean age of participants was 25.150 \pm 12.967 years.

Table (1): Demographic data of the patients.

\begin{tabular}{lc}
\hline Characteristics of patients & Mean \pm SD or N (\%) \\
\hline Age, years & $25.150 \pm 12.967$ \\
TBSA (\%) & $13.200 \pm 6.187$ \\
Gender: & $11(55 \%)$ \\
$\quad$ Male & $9(45 \%)$ \\
$\quad$ Female & \\
Site of STSG: & $18(90 \%)$ \\
$\quad$ Front of thigh & $2(10 \%)$ \\
$\quad$ Back of thigh &
\end{tabular}

TBSA: Total body surface area. STSG: Split-thickness skin graft.

It was noticed that healing days in donor site injected with PRF were lower than those in control site with statistical significance $(11.55 \pm 1.39$ days vs $13.45 \pm 1.70$ days; $p$-value $<0.001$ ) (Fig. 1 ). 
The mean epidermal thickness in this study was $113.55 \pm 3.62 \mu \mathrm{m}$ in the control group and $195.79 \pm 26.44 \mu \mathrm{m}$ in the PRF group, with statistical significance ( $p$-value <0.001) (Fig. 2).

Fig. (3) shows the clinical difference between PRF injected donor site and control site on day 10 and day 14 post-surgery with complete healing of the PRF injected donor area.

Regarding histological results in H\&E-stained sections, the epidermis was thicker on the PRFtreated side than on the control side. The control

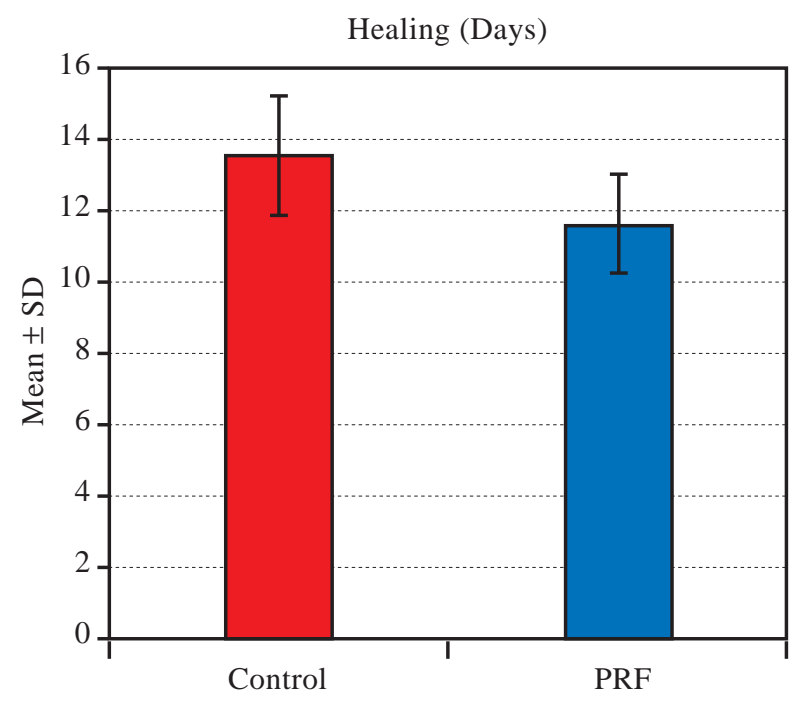

Fig. (1): Healing time in both PRF and control in days.

Case (1)

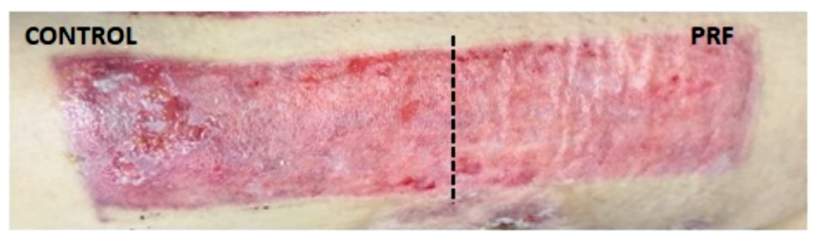

Case (2)

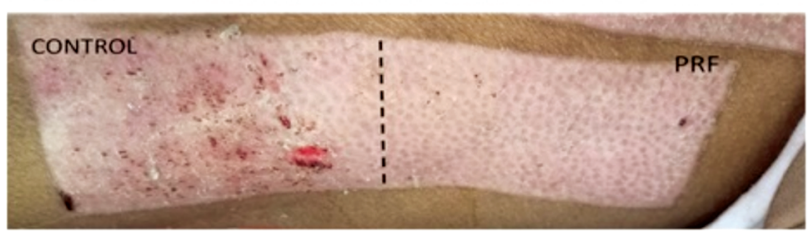

Day 10 side showed separation between the dermis and epidermis with an ill-defined basement membrane between both. Keratinocytes contained atypical cells with shrunken pyknotic nuclei and vacuolated cells were seen. While on the PRF-treated side the epidermis appeared normal. In Masson's trichromestained sections, the papillary dermis on the control sideshowed few fragmented and widely separated collagen fibers compared to the PRF-treated side. The reticular dermis contained thick collagen bundles. In orcein sections, minimal elastic fibers were seen in the papillary dermis on the control side compared to the PRF-treated side (Fig. 4).

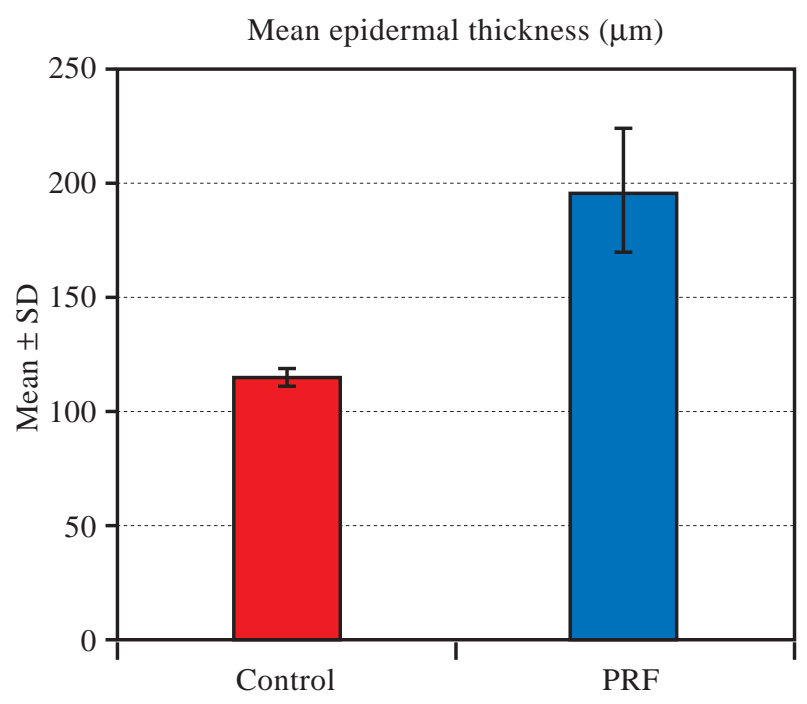

Fig. (2): Mean epidermal thickness in both PRF and control group in $\mu \mathrm{m}$.
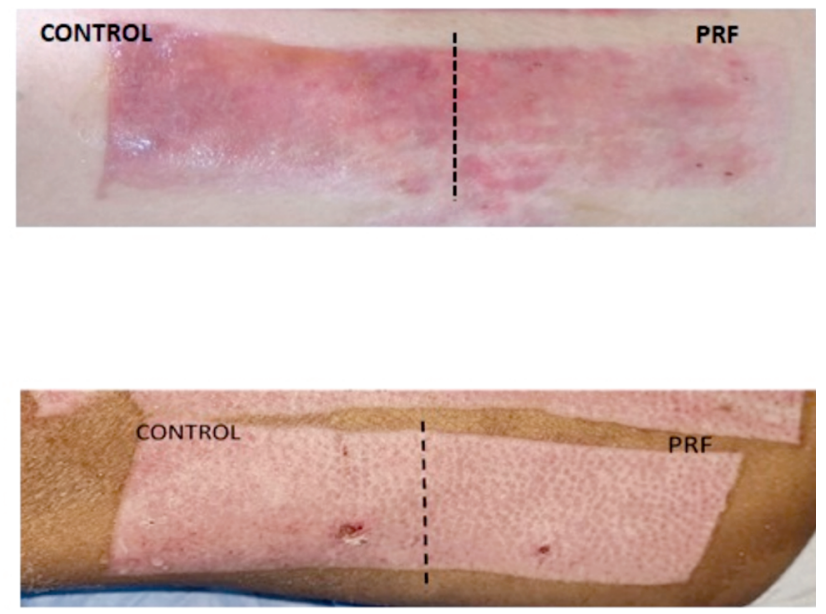

Day 14

Fig. (3): Clinical results for 2 patients at day 10 and day 14 post-surgery, the donor site was divided into two halves (dotted line) and the left and right-sided were designated the control and PRF-treated sides, respectively. 

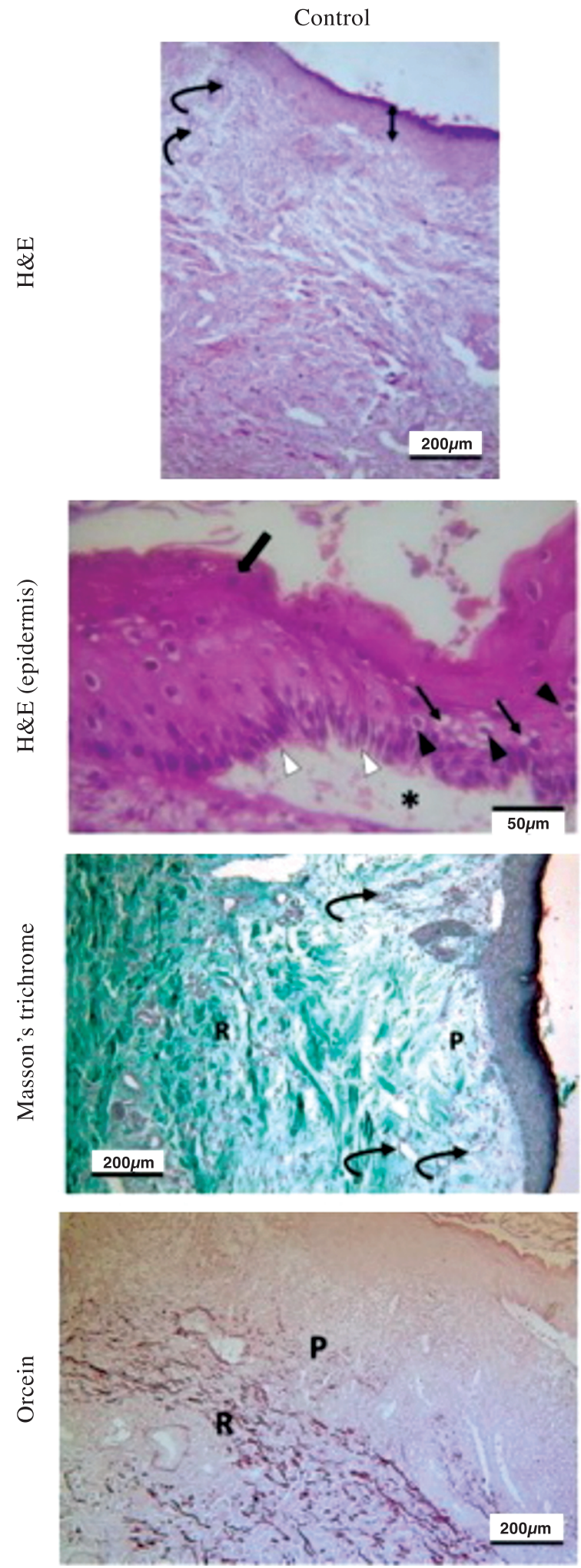

PRF
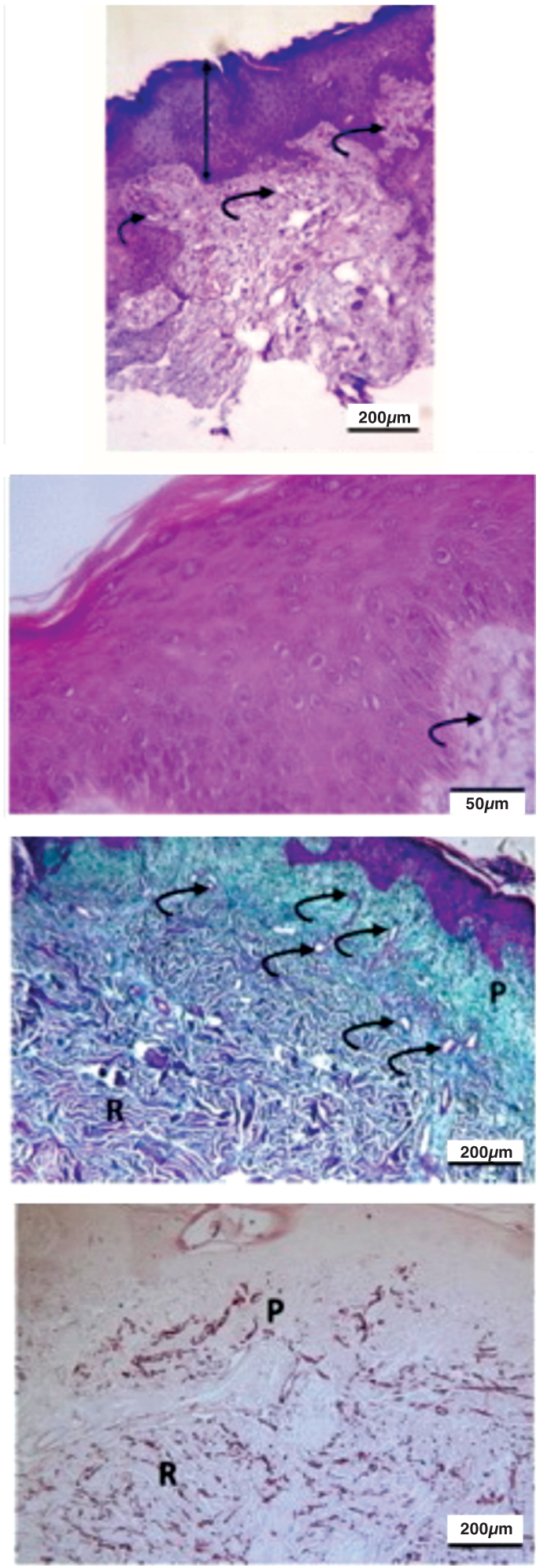

Fig. (4): Specimens from the control side and PRF treated side after hematoxylin and eosin (H\&E), Masson's trichrome, and orcein staining. In H\&E-stained sections, the epidermis $(\uparrow)$ is thicker on the PRF-treated side than on the control side. The epidermis of the control side shows separation (*) between dermis and epidermis. The epidermis is seen resting on an ill-defined basement membrane $(\Delta)$. Keratinocytes contain atypical cells $(\boldsymbol{\Delta})$ with shrunken pyknotic nuclei. Vacuolated cells $(\uparrow)$ are also seen. Notice the presence of nucleated cells in the stratum corneum (thick arrow). The epidermis of the PRF-treated side appears normal and the dermis contain numerous blood vessels (curved arrow). In Masson's trichrome sections few collagen fibers are seen in the papillary dermis $(\mathrm{P})$ on the control side compared to the PRF-treated side. In orcein sections, minimal elastic fibers are seen in the papillary dermis $(\mathrm{P})$ on the control side compared to the PRF-treated side. The reticular dermis (R) contains thick collagen bundles and elastic fibers. 


\section{DISCUSSION}

The current study showed that using injectable PRF on split-thickness skin graft donor areas resulted in a faster healing time than using topical conventional treatment (Vaseline gauze). Several studies found that applying platelet concentrates as a gel to the donor sites of STSG was superior to conventional treatment for wound healing. These studies looked at the effect of treatment modality on healing time, pain, itching, scarring, infection, and treatment complications $[\mathbf{2 , 9 , 1 0 ]}$.

Initial findings from our study revealed that the time it took for donor wounds to heal in PRF injected areas was shorter than in non-injected areas. The following are some possible explanations for our findings: Eliminating patients with comorbidities that may interfere with the healing process such as liver cell failure and heart failure, using the same patient donor area to compare between injected PRF area and control area, and thus many possible confounders were removed and histological assessment can provide a more precise assessment than prior researches.

Vaheb et al., studied the impact of applying PRF gel in donor sites of STSG. According to the findings of their study, the time and rate of healing of donor site of STSG wound were better in PRF group than in control group on the eighth and fifteenth days after surgery [9].

Fang et al., compared the impact of PRP gel versus traditional dressing on STSG wound healing; the rate of healing and recovery timewas better in PRF compared to the control group $(p<.05)$. One of their study's limitations is that it compares two groups with various wounds, which may result in numerous confounders in their study [11].

Our findings arenot supported by the findings of Danielsen et al., who found that the effect of PRF therapy on donor wound epithelialization was not significant [2]. The following are possible explanations for these findings: Examining foot ulcers caused by various etiologies andinability to conduct longer follow-up periods.

When compared to the previous management for donor wounds, PRF had a faster rate of wound healing (taking 10 to 14 days), whereas other authors reported treatment days ranging from 14 to 21 days $[\mathbf{9 , 1 2 , 1 3 ]}$.

As regards PRF's mode of action, It is worth noting that PRF is more beneficial in wound management as it contains many leucocytes that aid in the continuous release of growth factors.PRF gen- erates a significant amount of cytokines and has a platelet concentration four times that of blood [14-16].

In terms of histological evaluation; epithelization and keratinization of donor sites were observed in both untreated (control) and PRF-injected areas in the current study. Down growth of epidermis was noticed in PRF injected site. This down growth might be the beginning of the differentiation of epidermal cells from hair follicles.

In the current study, numerous blood vessels were identified in H\&E and Masson's trichromestained sections of the PRF injected site, which was consistent with previous researches [17-21]. The high levels of vascular endothelial growth factor (VEGF) and platelet-derived angiogenesis factor (PDAF) released by PRF could be attributed to angiogenesis [17]. Angiogenesis promotes vascular permeability during wound healing. The permeability of the arteries allows various cytokines and growth factors to reach the injured area and stimulate angiogenic activity [18]. It has been observed that newly formed blood vessels could provide nutrients and oxygen which help collagen synthesis [21].

In our study, mononuclear inflammatory cells were found in PRF-injected areas versus the untreated donor site which was in line with results discovered by other authors $[\mathbf{1 7 , 1 8}]$. In addition, mature thick collagen fibers were seen well organized in the dermis at the PRF-injected donor site, in contrast to the control donor location, where immature collagen fibers were commonly visible and this was in line with the findings of other researchers [17,18,21,22].

\section{Limitations and Strengths:}

The concentrations of growth factors and platelets were not measured by laboratory tests, and we were unable to conduct longer follow-up periods. Onthe other side, the study's greatest significant strength was using the samepatient's donor area to compare the efficacy of PRF. Also, we performed ahistological assessment which is mainly important in measuring mean epidermal thickness as well as the amount and quality of collagen fibers.

\section{Conclusion:}

When compared to traditional therapy, the outcomes of this study revealed that PRF can considerably minimize the time necessary for donor wound healing. More research on PRF for the treatment of different wounds of various etiologies, as well as longer follow-up periods, is recommended. 


\section{REFERENCES}

1- Reksodiputro M.H., Harba'i H.M., Koento T. and Harahap A.R.: Platelet-rich fibrin enhances wound epithelialization in the skin graft donor site. Journal of Physics: Conference Series, 1073: 032046, 2018.

2- Danielsen P., Jørgensen B., Karlsmark T., Jorgensen L.N. and Ågren M.S.J.P.: Surgery R. Effect of topical autologous platelet-rich fibrin versus no intervention on epithelialization of donor sites and meshed split-thickness skin autografts: A randomized clinical trial, 122 (5): 1431-40, 2008.

3- Hotwani K. and Sharma K.: Platelet-rich fibrin-a novel acumen into regenerative endodontic therapy. Restorative Dentistry \& Endodontics, 39 (1): 1-6, 2014.

4- Somani A. and Rai R.: Comparison of the efficacy of autologous platelet-rich fibrin versus saline dressing in chronic venous leg ulcers: A randomized controlled trial. Journal of Cutaneous and Aesthetic Surgery, 10 (1): 8, 2017.

5- Sclafani A.P.: Safety, efficacy, and utility of platelet-rich fibrin matrix in facial plastic surgery. Archives of Facial Plastic Surgery, 13 (4): 247, 2011.

6- Singh A., Kohli M. and Gupta N.: Platelet-rich fibrin: A novel approach for osseous regeneration. Journal of Maxillofacial and Oral Surgery, 11 (4): 430-4, 2012.

7- Miron R.J., Fujioka-Kobayashi M., Hernandez M., Kandalam U., Zhang Y., Ghanaati S., et al.: Injectable plateletrich fibrin (i-PRF): Opportunities in regenerative dentistry? Clinical Oral Investigations, 21 (8): 2619-27, 2017.

8- Suvarna S. and Layton C.: Bancroft's theory and practice of histological techniques, $7^{\text {th }}$ ed. Churchill Livingstone Elsevier. Oxford, 2013.

9- Vaheb M., Karrabi M., Khajeh M., Asadi A., Shahrestanaki E. and Sahebkar M.J. Tijolew: Evaluation of the effect of platelet-rich fibrin on wound healing at split-thickness skin graft donor sites: A randomized, placebo-controlled, triple-blind study, 20 (1): 29-36, 2021.

10- Kakudo N., Kushida S., Minakata T., Suzuki K. and Kusumoto K.J. Mmm: Platelet-rich plasma promotes epithelialization and angiogenesis in a split-thickness skin graft donor site, 44 (4): 233-6, 2011.

11- Fang Z., Yang X., Wu G., Liu M., Han J., Tao K., et al.: The use of autologous platelet-rich plasma gel increases wound healing and reduces scar development in splitthickness skin graft donor sites. Journal of plastic surgery and hand surgery, 53 (6): 356-60, 2019.

12- Ferreira L.M., Blanes L., Gragnani A., Veiga D.F., Veiga F.P., Nery G.B., et al.: Hemicellulose dressing versus rayon dressing in the re-epithelialization of split-thickness skin graft donor sites: A multicenter study, 18 (3): 88-94, 2009.

13- Kaiser D., Hafner J., Mayer D., French L.E. and Läuchli S.J. Ais: Care W. Alginate dressing and polyurethane film versus paraffin gauze in the treatment of split-thickness skin graft donor sites: A randomized controlled pilot study, 26 (2): 67-73, 2013.

14- Dohan Ehrenfest D.M., de Peppo G.M., Doglioli P. and Sammartino G.J.G.F.: Slow-release of growth factors and thrombospondin-1 in Choukroun's platelet-rich fibrin (PRF): A gold standard to achieve for all surgical platelet concentrates technologies, 27 (1): 63-9, 2009.

15- Dohan Ehrenfest D.M.J.O.S.: Oral medicine, oral pathology, oral radiology, endodontics. How to optimize the preparation of leukocyte-and platelet-rich fibrin (L-PRF, Choukroun's technique) clots and membranes: Introducing the PRF Box., 110 (3): 275-8, 2010.

16- Dohan D.M., Choukroun J., Diss A., Dohan S.L., Dohan A.J., Mouhyi J., et al.: Platelet-rich fibrin (PRF): A secondgeneration platelet concentrate. Part I: Technological concepts and evolution. Oral surgery, oral medicine, oral pathology, oral radiology, and endodontics, 101 (3): e3744, 2006.

17- Khanzadeh Alishahi M., Kazemi D., Mohajeri D., Mofidpoor H., Afkhami Goli A., Khanzadeh Alishahi MAJIJoVS, et al.: Histopathological evaluation of the effect of plateletrich fibrin on canine cutaneous incisional wound healing, 5 (2): 19-32, 2013.

18- Majeed A.A. and Abood DAJVW.: Histological assessment of the efficiency of rabbit serum in healing skin wounds, 12 (10): 1650, 2019.

19- Del Amo C., Perez-Valle A., Perez-Zabala E., Perez-delPecho K., Larrazabal A., Basterretxea A., et al.: Wound Dressing Selection Is Critical to Enhance Platelet-Rich Fibrin Activities in Wound Care, 21 (2): 624, 2020.

20- Tsai H-C., Chang GR-L., Fan H-C., Ou-Yang H., Huang L-C., Wu S-C., et al.: A mini-pig model for evaluating the efficacy of autologous platelet patches on induced acute full-thickness wound healing, 15 (1): 1-13, 2019.

21- Xu F., Zou D., Dai T., Xu H., An R., Liu Y., et al.: Effects of incorporation of granule-lyophilized platelet-rich fibrin into polyvinyl alcohol hydrogel on wound healing, 8 (1): 1-10, 2018.

22- Khalaf ${ }^{1}$ F.H. and SALIH SIJAJPCR: Clinical and histopathological evaluation of using platelet-rich plasma and platelet-rich fibrin matrix in the treatment of induced chronic open wounds in bucks, 11 (5): 337-41, 2018. 